\title{
Distance-Hereditary Embeddings of Circulant Graphs
}

\author{
Carmen Martínez, Ramón Beivide \\ Universidad de Cantabria \\ Dept. Electrónica y Computadores \\ Los Castros s/n. 39005. Santander. Spain \\ \{carmenmf,mon\}@atc.unican.es \\ Jaime Gutierrez \\ Universidad de Cantabria \\ Dept. Matemáticas y Computación \\ Los Castros s/n 39005. Santander. Spain \\ jaime@matesco.unican.es \\ Cruz Izu \\ The University of Adelaide \\ Dept. Of Computer Science \\ Adelaide, SA 5005. Australia \\ cruz@cs.adelaide.edu.au
}

\begin{abstract}
In this paper we present a distance-hereditary decomposition of optimal chordal rings of $2 k^{2}$ nodes into a set of rings of $2 k$ nodes, where $k$ is the diameter. All the rings belonging to this set have the same length and their diameter corresponds to the diameter of the chordal ring in which they are embedded. The members of this embedded set of rings are non-disjoint and preserve the minimal routing of the original circulant graph. Besides its practical consequences, our research allows the presentation of these optimal circulant graphs as a particular evolution of the traditional ring topology.
\end{abstract}

Key words.- Circulant graphs, Chordal Rings, Cycles, Ádam's Conjecture, Graph Embedding.

\section{Introduction}

Circulant graphs have deserved significant attention in the last decades. From a theoretical point of view, there are thousands of publications analyzing their algebraic properties. From a more practical perspective, circulant graphs have been employed in several applications. In the sixties, these graphs were used to build interconnection networks for distributed and parallel systems [8], [6]. In the seventies, circulant graphs constituted the basis for designing certain data alignment networks for com- plex memory systems [9]. In the eighties, several optimizations related to the diameter minimization of degree four circulant graphs, enhancing their applicability to the design of efficient interconnection networks [5], [4]. Nowadays, the analysis and characterization of circulant graphs and their applications still constitute active research areas.

A class of circulant graphs of degree four, with minimal topological distances, was presented in [2] as a basis for building interconnection networks for parallel computers. One of these graphs exists for any given number of nodes, which is defined by means of a single parameter. These graphs, denoted as midimew networks, are optimal because they have the minimum average distance among all circulant graphs of degree four; consequently, their diameters are also minimal. In addition, these graphs are regular, vertex-symmetric, maximally connected and, after an adequate transformation, they can be represented as mesh-connected topologies.

Several applications in computer science and engineering suggest the study of graph embedding techniques as a fundamental prerequisite to obtaining fast solutions to computational problems modelled as graphs or networks. In this paper we will consider graph embeddings in undirected chordal rings of degree four. As is well known, the structure of these graphs consists of an undirected ring in which a constant-length chord is added to every node in the ring. All the chordal rings consid- 
ered here are optimal as they have minimum average distance and diameter. Although all these optimal chordal rings have minimum distance-related properties, we will show that just half of them are isomorphic to midimew networks.

The main outcome of this research is a distancehereditary decomposition of optimal chordal rings into a set of rings. All the rings belonging to this set have the same length and their diameter corresponds to the diameter of the chordal ring in which they are embedded. The members of this embedded set of rings are non-disjoint and preserve the minimal routing of the original circulant graph. Besides its practical consequences, our research allows the presentation of these optimal circulant graphs as a particular evolution of the traditional ring topology.

The rest of this paper is organized as follows: Section 2 is devoted to introducing some notation and to presenting the decomposition of certain optimal circulants of degree four into circulants of degree two. In section 3, we introduce a new class of optimal chordal rings. Section 4 is dedicated to establishing a new distance-hereditary decomposition of such chordal rings. Finally, section 5 summarizes the main conclusions of this research.

\section{Dense Optimal Circulants of Degree Four}

For a self contained paper, we are going to introduce here some notation, definitions and known results, for later use.

A circulant graph with $N$ vertices and jumps $j_{1}, j_{2}, \ldots, j_{m}$ is an undirected graph in which each vertex $n, 0 \leq n \leq N-1$, is adjacent to all the vertices $n \pm j_{i} \bmod N$, with $1 \leq i \leq m$. We denote this graph as $C_{N}\left(j_{1}, j_{2}, \ldots, j_{m}\right)$. The family of circulant graphs includes the complete graph and the cyclic graph or ring among its members.

We say that a circulant is dense if it has the maximum possible number of nodes for a given diameter. Thus, if $k$ is a positive integer, a dense ring or $C_{N}(1)$ of degree two has $2 k+1=k+(k+1)$ nodes. Conversely, the non-dense ring has $2 k=$ $k+k$ nodes. When adding another jump to the list, every integer $k$ defines a family of $4 k$ optimal $C_{N}\left(j_{1}, j_{2}\right)$ graphs or midimew networks of diameter $k$, with $j_{1}=b-1$ and $j_{2}=b$, where $b=$ $\left\lceil\sqrt{\frac{N}{2}}\right\rceil$, see [2]. The dense graph, $C_{N}(k, k+1)$, contains $2 k^{2}+2 k+1$ nodes. The other graphs in the family correspond to non-dense values of $N$. In the following sections we will focus our attention on optimal non-dense $C_{N}\left(j_{1}, j_{2}\right)$ graphs in which $N=2 k^{2}=k^{2}+k^{2}$.

In [3] it has been shown how dense $C_{N}(k, k+1)$ graphs or midimew networks can be decomposed into a non-disjoint collection of $2 N$ rings that preserves the distance-related properties of the original graph. These rings, or circulants of degree two, are also dense and their diameter is the diameter of the circulant in which they are embedded. In short, a dense $C_{N}(k, k+1)$, with $N=2 k^{2}+2 k+1=$ $k^{2}+(k+1)^{2}$, can be decomposed into a set of $2 N$ rings, each of them having $2 k+1=k+(k+1)$ nodes. Every vertex is present in $2(2 k+1)$ rings. It is straightforward to prove that if $C_{N}\left(j_{1}, j_{2}\right)$ and $C_{N}\left(i_{1}, i_{2}\right)$ are two dense optimal circulants, then $C_{N}\left(j_{1}, j_{2}\right)$ is isomorphic to $C_{N}\left(i_{1}, i_{2}\right)$, that is, $C_{N}\left(j_{1}, j_{2}\right) \cong C_{N}\left(i_{1}, i_{2}\right)$. Consequently, every dense optimal circulant of degree four can be decomposed into the same distance-hereditary set of dense rings, which can be calculated by the graph isomorphism mapping $C_{N}(k, k+1)$ onto this one. Now we introduce the Ádam's Conjecture, see [1], which it is known to be a theorem for circulants of degree four, see [7]. We are using it for finding out the isomorphism between the graphs $C_{N}(k, k+1)$ and $C_{N}(1,2 k+1)$. As usual, we denote by $\mathcal{U}\left(\mathbb{Z}_{N}\right)$ the group of the units of the ring $\mathbb{Z}_{N}$ (the ring of the integers modulo $N)$, that is, $\mathcal{U}\left(\mathbb{Z}_{N}\right)=\{u \in$ $\left.\mathbb{Z}_{N} \mid \operatorname{gcd}(u, N)=1\right\}$.

Theorem 1 Let $N$ be a natural number. We have, $C_{N}\left(j_{1}, j_{2}\right) \cong C_{N}\left(i_{1}, i_{2}\right) \Leftrightarrow \exists u \in$ $\mathcal{U}\left(\mathbb{Z}_{N}\right) \mid u\left\{ \pm j_{1}, \pm j_{2}\right\}=\left\{ \pm i_{1}, \pm i_{2}\right\} \bmod N$.

Hence, the element $u=(k+1)^{-1} \in \mathcal{U}\left(\mathbb{Z}_{N}\right)$ provides the adequate isomorphism between the graphs $C_{N}(k, k+1)$ and $C_{N}(1,2 k+1)$, where $N=2 k^{2}+2 k+1$. Consequently, for every natural number $k>1$ the optimal graphs $C_{N}(1,2 k+1)$ also have a distance-hereditary non-disjoint decomposition into $2 N$ rings of length $2 k+1$.

Finally, it is interesting to remark that we can see a dense $C_{N}(1,2 k+1)$ chordal ring graph, with $N=k^{2}+(k+1)^{2}$, as a particular evolution of the dense $C_{N}(1)$ graph, with $N=k+(k+1)$.

\section{Optimal Non-Dense Chordal Rings of Degree Four}

In this section we present a new class of optimal circulants of degree four, with $N=2 k^{2}$ nodes. 
The following proposition provides an optimal chordal ring with this number of nodes, which was not covered by the ones presented in [2].

Proposition 2 Let $k>1$ be an integer number. The graph $C_{2 k^{2}}(1,2 k-1)$ is optimal.

Sketch of the Proof.- In order to show that $C_{2 k^{2}}(1,2 k-1)$ has minimum average distance, it suffices to prove that the mapping $f: D_{k-1} \longrightarrow$ $\mathbb{Z}_{2 k^{2}}$ defined as $f(x, y)=x+(2 k-1) y \bmod 2 k^{2}$ is injective, where $D_{k-1}=\{(x, y) \in \mathbb{Z} \times \mathbb{Z}|| x \mid+$ $|y| \leq k-1\}$. Suppose $f(x, y)=f\left(x^{\prime}, y^{\prime}\right)$ with $(x, y),\left(x^{\prime}, y^{\prime}\right) \in D_{k-1}$. We have, $x+(2 k-1) y=$ $x^{\prime}+(2 k-1) y^{\prime} \bmod 2 k^{2}$ and there exists $\alpha \in \mathbb{Z}$ such that $\left(x-x^{\prime}\right)+(2 k-1)\left(y-y^{\prime}\right)=2 \alpha k^{2}$. Taking absolute values on both sides of the previous equality we get $2 k^{2}|\alpha| \leq 4 k(k-1)$. Hence $|\alpha| \leq 2-\frac{2}{k} \leq 1$, that is, $\alpha \in\{-1,0,1\}$. Analyzing the three cases, we obtain that the unique solution is $\alpha=0$. Hence, the only possibility is $x=x^{\prime}, y=y^{\prime}$. Consequently the injectivity of the mapping $f(x, y)$ has been proved.

Next we will show that the chordal ring defined in Proposition 2 is really new, that is, it is not isomorphic to the corresponding $C_{N}(b-1, b)$ midimew graph, where $N=2 k^{2}$.

Theorem 3 Let $k$ be a natural number. The graphs $C_{2 k^{2}}(1,2 k-1)$ and $C_{2 k^{2}}(k-1, k)$ are not isomorphic.

Sketch of the Proof.- Suppose the contrary, that is, $C_{2 k^{2}}(1,2 k-1)$ and $C_{2 k^{2}}(k-1, k)$ are isomorphic. By Theorem 1 , there exits $u \in \mathcal{U}\left(\mathbb{Z}_{2 k^{2}}\right)$, such that:

$u\{ \pm 1, \pm(2 k-1)\}=\{ \pm(k-1), \pm k\} \bmod 2 k^{2}$.

Obviously $k \notin \mathcal{U}\left(\mathbb{Z}_{2 k^{2}}\right)$ and $\operatorname{gcd}\left(2 k^{2},-4 k+1\right)=$ 1 , it shows a contradiction.

\section{Ring embedding in Non-dense Opti- mal Chordal Rings}

In this section we compute explicitly a decomposition of $C_{N}(1,2 k-1)$, where $N=2 k^{2}$, into $2 N$ rings with $2 k$ nodes and preserving the minimal routing of the original graph.

In order to obtain these kind of rings without repeating cases in our study, we only consider rings of $2 k$ nodes obtained taking positive steps in jumps $\{1,2 k-1\}$ or taking positive steps in jumps $\{1,-(2 k-1)\}$. Therefore, for the rest of the paper, a $2 k$-ring constructed from node $n_{1}$ in $C_{2 k^{2}}(1,2 k-1)$ is a cycle such that, if $\left\{n_{1}, n_{2}, \ldots, n_{2 k}\right\}$ is its set of nodes, then it must fulfill one of these two conditions:

i) $n_{i+1}=n_{i}+1 \bmod 2 k^{2}$ or $n_{i+1}=n_{i}+$ $(2 k-1) \bmod 2 k^{2}, \forall i \in\{1,2, \ldots, 2 k\}$

ii) $n_{i+1}=n_{i}+1 \bmod 2 k^{2}$ or $n_{i+1}=n_{i}-$ $(2 k-1) \bmod 2 k^{2}, \forall i \in\{1,2, \ldots, 2 k\}$.

Although in the dense case all the optimal circulants of degree four have a decomposition into dense rings, in this case there are optimal circulants that contain rings whose sizes are different to $2 k$. As an example, when $N=18$, it can be seen that $C_{N}(b-1, b)=C_{18}(2,3)$ contains rings with 7 nodes.

Next, we will show that the optimal graph defined in Proposition 2 can be decomposed into $2 \mathrm{~N}$ $2 k$-rings. As a first step we take a local approach of the problem: we obtain all the $2 k$-rings from node 0 . By node symmetry it suffices to consider the $2 k$-rings constructed from a given node, so we have chosen node 0 for simplicity.

We need the following technical and elementary result:

Lemma 4 Let $k$ be a positive integer. Consider the diophantine linear systems in the unknowns $(x, y, \alpha)$ :

$$
\begin{aligned}
& (S 1)\left\{\begin{array}{c}
x+(2 k-1) y-2 k^{2} \alpha=0 \\
x+y=2 k
\end{array}\right. \\
& (S 2)\left\{\begin{array}{c}
x-(2 k-1) y-2 k^{2} \alpha=0 \\
x+y=2 k
\end{array}\right.
\end{aligned}
$$

We have,

i) $(x, y, \alpha)=(k, k, 1)$ is the unique solution of $(S 1)$ verifying $x, y \geq 0$.

ii) $(x, y, \alpha)=(k-1, k+1,-1),(2 k-1,1,0)$ are the unique solutions of $(S 2)$ verifying $x, y \geq 0$.

In order to simplify the proof of the remaining results, we introduce the following notation. 
Notation 5 We represent a $2 k$ - ring as a vector whose coordinates are in the set $\{0,1\}$ or in $\{0,-1\}$, depending on the type of $2 k-$ ring. If we are considering a $2 k$-ring in $C_{2 k^{2}}(1,2 k-1), 0$ represents a (positive) step in the jump 1,1 represents a step in $2 k-1$ and -1 a step in $-(2 k-1)$. For example, the 6-tuple $(0,1,0,1,0,1)$ denotes the 6 -ring $\{0,1,6,7,12,13\}$ in $C_{18}(1,5)$.

Proposition 6 Let $k$ be a positive integer. Then, any $2 k$-ring from node 0 in $C_{2 k^{2}}(1,2 k-1)$ belongs to one of the following sets:

$A_{1}=\left\{\left(x_{1}, \ldots, x_{2 k}\right) \mid x_{i}=0,1, \sum x_{i}=k\right\}$

$A_{2}=\left\{\left(x_{1}, \ldots, x_{2 k}\right)\left|x_{i}=0,-1, \sum\right| x_{i} \mid=k+1\right\}$

$A_{3}=\left\{\left(x_{1}, \ldots, x_{2 k}\right)\left|x_{i}=0,-1, \sum\right| x_{i} \mid=1\right\}$

There are $\left(\begin{array}{c}2 k \\ k\end{array}\right)+\left(\begin{array}{c}2 k \\ k+1\end{array}\right)+\left(\begin{array}{c}2 k \\ 1\end{array}\right)$ $2 k$-rings from node 0 in $C_{2 k^{2}}(1,2 k-1)$.

Sketch of the Proof.- We observe that the solutions of Lemma 4 are the total number of steps allowed to construct a $2 k$-ring, since

$x+(2 k-1) y-2 k^{2} \alpha=0 \Rightarrow x+(2 k-1) y=$ $0 \bmod 2 k^{2}$ and $x-(2 k-1) y-2 k^{2} \alpha=0 \Rightarrow$ $x-(2 k-1) y=0 \bmod 2 k^{2}$.

The proof ends just counting the cardinal of the previous three sets.

Next, we compute a subset of the set $A_{1} \cup A_{2}$. We will show the cardinality of this set is $2 k-1$ and, every node is reached from node 0 with at least one of the rings of this selection.

Theorem 7 With the above notation, we consider the following sets of $2 k$-rings:

i) $A_{1}^{k}=\left\{\bar{\lambda}_{1}, \ldots, \bar{\lambda}_{k-1}\right\} \subset A_{1}$ where $\bar{\lambda}_{i}=$ $(\overbrace{0, \ldots, 0}^{i}, \overbrace{1, \ldots, 1}^{k}, \overbrace{0, \ldots, 0}^{k-i})$, for $i=$ $1,2, \ldots, k-1$.

ii) $A_{2}^{k}=\left\{\bar{\mu}_{1}, \ldots, \bar{\mu}_{k}\right\} \subset A_{2}$ where $\bar{\mu}_{j}=$ $(\overbrace{-1, \ldots,-1}^{j}, \overbrace{0, \ldots, 0}^{k-1}, \overbrace{-1, \ldots,-1}^{k-j+1})$, for $j=1,2, \ldots, k$.

We have that for every node $n$ of $C_{2 k^{2}}(1,2 k-1)$ there exists an element in $A^{k}=A_{1}^{k} \cup A_{2}^{k}$ containing $n$.

Sketch of the Proof.- Given a node $n$, we have $n=$ $x(2 k-1)+y \bmod 2 k^{2}$, for some $(x, y) \in D_{k}$, where $D_{k}=\{(x, y) \in \mathbb{Z} \times \mathbb{Z}|| x|+| y \mid \leq k\}$.
Hence, $|x|,|y| \leq k$. We distinguish four cases: $x=k, 0<x<k, x=0$ and $0 \leq x \leq k$. In order to illustrate the proof we only consider the following case: $0<x<k$.

- If $y=0$ then $n=x(2 k-1) \bmod 2 k^{2}=$ $(k+1-x)(-(2 k-1))+(k-1) \bmod 2 k^{2}$. As $0<k+1-x \leq k$ then $n \in \bar{\mu}_{k+1-x}$.

- If $0<y<k$ then $n \in \bar{\lambda}_{y}$.

- If $|y|=k$ then $x=0$, so this case is not possible.

- If $-k<y<0$ then $n=x(2 k-1)+$ $y \bmod 2 k^{2}=(k+1-x)(-(2 k-1))+(k-1+$ y) $\bmod 2 k^{2}$. Observe that $0 \leq(k-1)+y \leq k$ and $2 \leq k+1-x \leq k$. Then, $n \in \bar{\mu}_{k+1-x}$.

So, $n$ belongs to a $2 k-$ ring of $A^{k}=A_{1}^{k} \cup A_{2}^{k}$.

Finally, we consider the union of subsets of $2 k$-rings for all the nodes of the graph. We will show that this union has $2 N$ different elements. So, we will have decomposed the graph into a set of $2 N 2 k$-rings, providing a complete picture of the graph.

We denote by $A^{k}(n)$ the set of $2 k$-rings with $2 k-1$ elements constructed as we have done for node 0 in Theorem 7, but for node $n$. Now, we are considering the whole set of $2 k$-rings

$$
B^{k}=\bigcup_{n=0}^{N-1} A^{k}(n)
$$

Obviously, for every pair of nodes $n, m$ there exists a $2 k-$ ring in $B^{k}$ that contains them. Now, we study the cardinal of this set.

Theorem 8 Let $k$ be a positive integer and $N=$ $2 k^{2}$. Then, the cardinal of $B^{k}$ is $2 N$.

Sketch of the Proof.- We consider the sets

$$
B_{1}^{k}=\bigcup_{n=0}^{N-1} A_{1}^{k}(n), \quad B_{2}^{k}=\bigcup_{n=0}^{N-1} A_{2}^{k}(n)
$$

Then, $\operatorname{card}\left(B_{1}^{k}\right) \leq(k-1) N$ and $\operatorname{card}\left(B_{2}^{k}\right) \leq$ $k N$. It is clear that if there exists a $2 k$-ring $\alpha$ such that $\alpha \in A^{k}(n) \cap A^{k}(m)$, then either $\alpha \in A_{1}^{k}(n) \cap$ $A_{1}^{k}(m)$ or $\alpha \in A_{2}^{k}(n) \cap A_{2}^{k}(m)$ (where $n \neq m$ ), so we can analyze the cardinal of the sets $B_{1}^{k}$ and $B_{2}^{k}$ separately. Therefore, $\operatorname{card}\left(B^{k}\right)=\operatorname{card}\left(B_{1}^{k}\right)+$ $\operatorname{card}\left(B_{2}^{k}\right)$. Now, we show that every ring in $B_{1}^{k}$ 
is repeated $k-1$ times and every ring in $B_{2}^{k}$ is repeated $k$ times, so we obtain $\operatorname{card}\left(B^{k}\right) \leq N+$ $N=2 N$. In order to prove this last claim, it is enough to show this for each ring from node 0 .

First, we see that every ring in $\bar{\lambda}_{i} \in A_{1}^{k}(0)$ belongs to other $k-2$ sets of rings from other $k-2$ different nodes. In fact, let $n_{s}=s \bmod N$. We consider the following set of $k-1$ different nodes: $\left\{n_{-(k-1-i)}, \ldots n_{-1}, n_{0}, \ldots, n_{i-1}\right\}$. We have that $n_{0}=0$ and, since $1 \leq i \leq k-1$, this set of nodes is composed of $k-1$ different elements. Then, this ring appears once in $A_{1}^{k}\left(n_{s}\right)$ for $s=$ $-(k-1-i), \ldots,-1,0,1, \ldots i-1$. The transformation is done in the following way: $\bar{\lambda}_{i} \in A_{1}^{k}(0)$ becomes $\bar{\lambda}_{i-s} \in A_{1}^{k}\left(n_{s}\right)$.

Finally, we show that every ring in $\bar{\mu}_{j} \in A_{2}^{k}(0)$ belongs to other $k-1$ sets of rings from other $k-1$ different nodes. In fact, let $n_{t}=$ $t(-(2 t-1)) \bmod N$. We consider the set of $k$ different nodes $\left\{n_{-(k-j)}, \ldots n_{-1}, n_{0}, \ldots, n_{j-1}\right\}$. Again, $n_{0}=0$. Thus, $\bar{\mu}_{j} \in A_{2}^{k}\left(n_{t}\right)$, for $t=$ $-(k-j), \ldots,-1,0,1, \ldots j-1$, and the transformation is carried out, as in the previous case, translating the ring in the following way: $\bar{\mu}_{j} \in A_{2}^{k}(0)$ becomes $\bar{\mu}_{j-t} \in A_{2}^{k}\left(n_{t}\right)$, obtaining the $k-1$ repetitions.

Observe that the repetitions considered above are the only ones allowed, so we get the equality.

\section{Conclusions.}

In this paper, we have shown that some optimal chordal rings of degree four, or $C_{N}(1,2 k \pm 1)$ have a distance-hereditary non-disjoint decomposition into $2 N$ rings. This property can be successfully exploited to map a great number of parallel applications that can be solved in terms of rings. Moreover, this decomposition can also be useful for a balanced management of a parallel computer based on these circulant topologies under a policy of space and time sharing among different parallel tasks.

In addition, we can conclude that some members belonging to the family of optimal circulant graphs of degree four are a particular evolution of the traditional ring topology or circulants of degree two. In particular, for $k>1$, the non-dense ring or $C_{k+k}(1)$ graph has an image into the non-dense $C_{k^{2}+k^{2}}(1,2 k-1)$ optimal chordal ring and the dense ring or $C_{k+(k+1)}(1)$ graph has an image into the dense $C_{k^{2}+(k+1)^{2}}(1,2 k+1)$ optimal chordal ring. All these circulants of degree four can be seen as a homogeneous collection of $2 N$ rings of diameter $k$, which conserves the distance properties of the original graph.

Acknowledgments.- The first two authors are partially supported by the Spanish CICYT project TIC2001-0591-C02-01 and by the Spanish Ministry of Education under grant PR2002-0043. The third author is partially supported by the Spanish project BFM2001-1294.

\section{References}

[1] A. Ádám. Research problem 2-10. J. Combinatorial Theory, 393, 1109-1124, 1991.

[2] R. Beivide, E. Herrada, J.L. Balcázar and A. Arruabarrena. Optimal Distance Networks of Low Degree for Parallel Computers. IEEE Transactions on Computers, Vol. C-40, No. 10, pp. 1109-1124, 1991.

[3] R. Beivide, C. Izu, C. Martínez, J.A. Gregorio. Pythagorean Graphs: Suitable Topologies for Interconnection Networks. Submited. Also available at atc.unican.es/REPORTS/TR2002-56.pdf.

[4] J.-C. Bermond, F. Comellas and D.F. Hsu. Distributed Loop Computer Networks: A Survey. Journal of Parallel and Distributed Computing, Vol. 24, pp. 2-10, 1995.

[5] F.T. Boesch and J.F. Wang. Reliable Circulant Networks with Minimum Transmission Delay. IEEE Transactions on Circuits and Systems, Vol.33, No.12, pp.1286-1291. 1985.

[6] W.J. Bouknight, S.A. Denenberg, D.E. McIntyre, J.M. Randall, A.H. Sameh and D.L. Slotnick. The Illiac IV System. Proc. IEEE, Vol. 60, No. 4, pp. 369-388. 1972.

[7] B. Mans, F. Pappalardi and I. Shparlinski. On the Ádám Conjecture on Circulant Graphs. Discrete Math., 2002, v.254, 309-329.

[8] R. S. Wilkov. Analysis and Design of Reliable Computer Networks. IEEE Trans. Communications, Vol. 20, 660-678.1972.

[9] C.K. Wong and D. Coppersmith. A Combinatorial Problem Related to Multimodule Memory Organizations. Journal of the ACM, Vol. 21, No.3, 392-402. 1974. 